\title{
Chemical and Biological Characterization from Condalia microphylla Fruits, a Native Species of Patagonia
}

\section{Argentina}

\author{
Patricia Boeri $^{1}$, Lucrecia Piñuel ${ }^{1,2}$, Sandra Sharry ${ }^{1,3}$, Andrea Tombari ${ }^{1}$ and Daniel Barrio ${ }^{1,2}$ \\ 1. Departamento de Ciencias Exactas, Naturales y de Ingeniería, Sede Atlántica, Universidad Nacional de Río Negro, Don Bosco y \\ Leloir, Viedma 8500, Río Negro, Argentina \\ 2. Consejo Nacional de Investigaciones Científicas y Técnicas (CONICET), Sede Atlántica, Universidad Nacional de Río Negro \\ (UNRN), Av. Don Bosco, Viedma 8500, Río Negro, Argentina \\ 3. Facultad de Ciencias Agrarias y Forestales, Universidad Nacional de La Plata, Calle $60 \mathrm{~s} / \mathrm{n}$. La Plata, Buenos Aires 1900, \\ Argentina
}

\begin{abstract}
Condalia microphylla Cav. (Rhamnaceae), popularly known as "piquillin", is widely distributed in Patagonia. The drupes are consumed as fresh fruits by Argentine communities. The aim of this work was to quantify the nutritional value of C. microphylla fruit and the phenolic compounds present and to determine the functional antioxidant properties in vitro and in vivo. The nutritional value was determined according to the Association of Official Analytical Chemists (AOAC) methodology, and phenolic compounds were quantified by diode-array detection (HPLC-DAD). Antioxidant activity in vitro and in vivo was analyzed through the use of the radical species 2,2-diphenyl-1-picrylhydrazyl (DPPH) and zebrafish model, respectively. Quercetin-3-O-rutinoside (rutin) was the single principal phenolic compound. The extracts contained in vitro antioxidant activities and total phenolic contents (TPCs) between $1,143 \pm 112 \mu \mathrm{g}$ and 4,633 $\pm 174 \mu \mathrm{g}$ gallic-acid equivalents (GAEs) per $100 \mathrm{~g}$ dry weight (DW), though no relationship was found between the latter parameter and the antioxidant activity of the extracts. When zebrafish larvae were exposed to oxidative stress $\left(2.4 \% \mathrm{v} / \mathrm{v} \mathrm{H}_{2} \mathrm{O}_{2}\right)$, a concentration as low as $1.44 \mu \mathrm{g}$ of GAEs/mL of piquillin-derived polyphenols inhibited lipid oxidation by up to $40 \%$. Thus, in view of these advantageous functional food properties and the opportunity to exploit this Patagonian natural resource, piquillin consumption should be promoted worldwide.
\end{abstract}

Key words: Piquillin, antioxidant activity, zebrafish, in vivo activity.

\section{Introduction}

Piquillin (Condalia microphylla Cav.), a thorny evergreen rhamnaceous plant, is a dominant shrub of the Patagonian Monte semidesert. This native species provides wood, food and various active principles [1]. The fresh fruit of piquillin is widely consumed by many communities of Patagonia, Argentina, and for a long time has been used in many preparations of traditional medicine [1]. The fruits are a reddish-brown spherical drupe with a sticky, sweet

Corresponding author: Patricia Boeri, Ph.D., research fields: plant biotechnology and bioprospecting. pulp, and similar to those of Ziziphus, another genus in the Rhamnaceae family, has been extensively studied [2, 3]. Phytochemical investigations have revealed that Ziziphus fruits are rich sources in bioactive compounds with biologic properties potentially beneficial to human health, such as antiarrhythmic [4], antioxidant [5], antiseizure [6] and antitumor activities [7]. Many degenerative diseases are associated with an imbalance between prooxidants and antioxidants within the body, a phenomenon referred to as oxidative stress [8]. In recent decades, phytochemicals, such as flavonoids, catechins, tannins and anthocyanins, among other polyphenols, have 
attracted a growing attention, because their redox properties can prevent or delay the development of degenerative diseases [9]. These secondary metabolites, however, are often present in low quantities in plant material; and their extraction, purification and characterization still remain a major challenge in drug discovery and development. Therefore, the most appropriate extraction methodology and the most suitable analytical technique must be sought in order to achieve the best extraction, purification and final isolation of the desired secondary metabolite.

Numerous studies have confirmed that polyphenols exert a protective action on human health and are key components of a healthy, balanced diet. Epidemiological studies suggest that a high dietary intake of these compounds is associated with a reduced incidence of chronic ailments, such as cardiovascular disease, diabetes and cancer. In particular, a group of polyphenols known as flavonoids have been shown to exert a variety of anticarcinogenic effects, including an ability to induce apoptosis in tumor cells, inhibit cancer cell proliferation and prevent angiogenesis and tumor cell invasion [10]. An interest in the investigation of the biologic activities and bioactive compounds of fruits and vegetables has increased significantly in recent years. To the knowledge of the authors, no review has yet been published on the investigation of bioactive compounds in piquillin fruits and the potential benefits that might be attributed to $C$. microphylla, if extracts of the fruit were to be used for nutraceutical purposes.

The evaluation of the behaviour of a single compound or chemical mixture can be approached though the use of biological models in vitro or the in vivo activity in whole animals. The zebrafish model has been used as an alternative paradigm in ambits, such as the food and pharmaceutical industries in order to investigate the teratogenic, embryotoxic and genotoxic properties, as well as the beneficial embryologic and neurobiologic activities of very diverse substances in addition to other types of study [11]. The present work employed an in vivo model based on the zebrafish (Danio rerio), in which the biochemical parameters of embryos were analyzed to determine the antioxidant effects in vivo of extracts from the fruit of C. microphylla.

In environments, such as the Patagonia, Argentina where nutrient availability is very limited, the plants have developed a wide variety of metabolites as a result of both selective pressure and resource availability [12]. In view of these considerations involving the ongoing quest to find new edible plants with functional properties and to promote the reproduction and preservation of those taxa, the experiments were undertaken to be described here on the chemical and biologic properties of the fruit of $C$. microphylla - an area as yet unexplored. The aim of these investigations was to determine the nutritional composition of the fruit C. microphylla grown in the arid and semi-arid region from Patagonia, Argentina with an aim at characterizing the different bioactive phytocompounds having potential antioxidant properties.

\section{Materials and Methods}

\subsection{Chemical Reagents}

Folin-Ciocalteu reagent, 2,2-diphenyl-1picrylhydrazyl (DPPH), $\alpha$-tocopherol, gallic acid, dimethyl sulfoxide (DMSO), D-glucose, thiobarbituric acid (TBA) and Tween 20 were purchased from Sigma Chemical Co. (St Louis, MO, USA). The other chemicals used were of analytical grade.

\subsection{Samples}

\subsubsection{Plant Materials}

The fruits from at least 20 individuals of $C$. microphylla were collected during February in 2012, 2013 and 2014 in Northern Patagonia (41 ${ }^{\circ} 55^{\prime} 296^{\prime \prime}$ S, $\left.63^{\circ} 05^{\prime} 693^{\prime \prime} \mathrm{W}\right)$.

Ripe fruits were washed with distilled water and 
quickly processed to facilitate the separation of manually removed endocarps. The pulp thus obtained was stored at $-20{ }^{\circ} \mathrm{C}$ in the dark until use for analysis.

\subsubsection{Sample Processing}

Three types of pulp extracts obtained with the following three solvents were evaluated: (1) methanol;

(2) $70 \% \mathrm{v} / \mathrm{v}$ aqueous acetone; (3) $50 \% \mathrm{v} / \mathrm{v}$ aqueous DMSO. The pulp-to-solvent ratio was 1:10. In all preparations, the extraction was performed overnight at room temperature with shaking $(500 \mathrm{rpm})$, followed by centrifugation at $7,000 \mathrm{rpm}$ for $15 \mathrm{~min}$. For biologic activity determination, the acetone was removed from the extract in a vacuum oven at $40{ }^{\circ} \mathrm{C}$ and the residue resuspended in $5 \% \mathrm{v} / \mathrm{v}$ aqueous DMSO.

\subsection{Determination of Chemical Composition}

The measurement of ash, dietary fiber, protein and lipids was performed according to the methodology specified by the Association of Official Analytical Chemists (AOAC) [13].

Sugar determination: total carbohydrates were determined according to the volumetric method of Fehling-Causse-Bonnans [13]; total polysaccharides, expressed as glucose (Glu) equivalents, by the phenol-sulfuric acid method [14], and total soluble reducing sugars, expressed as $\mathrm{g}$ of glucose per $100 \mathrm{~g}$ dry weight (DW), by the method of Somogyi-Nelson [15].

\subsection{Total Phenolic Content}

The total phenolic content (TPC) of the fruit extracts was determined by the Folin-Ciocalteu colorimetric method described by Singleton et al. [16] with absorbance measurements at $760 \mathrm{~nm}$ in a spectrum SP-2100UV/SP spectrophotometer. The contents of total phenolic compounds in three extracts from the pulp of piquillin fruit were calculated with gallic acid as the standard. The measurement was performed in triplicate and TPC was expressed in $\mathrm{mg}$ of gallic-acid equivalents (GAEs) per $100 \mathrm{~g}$ DW.

\subsubsection{Determination of Polyphenols}

The determination of polyphenol composition was performed at the Center for Research and Technical Assistance to Industry (CIATI-AC), Villa Regina, Río Negro. The total ascorbic acid, ferulic acid, gallic acid, rutin (quercetin-3-O-rutinoside), $p$-coumaric acid, catechin and epicatechin were determined by high performance liquid chromatography with diode-array detection (HPLC-DAD) (Shimadzu).

\subsubsection{Quantification of Anthocyanins}

Total anthocyanin content-expressed as $\mathrm{mg}$ cyanidin-3-glucoside equivalents (C3GE) per $100 \mathrm{~g}$ DW - was evaluated by the differential-pH method [17].

\subsection{Antioxidant Activity}

\subsubsection{Antioxidant Activity in Vitro}

The pulp extracts from ripe fruit of C. microphylla were evaluated for antioxidant activity. The ability to capture free radicals by antioxidants was analyzed through the use of the radical species DPPH [18] by following the decrease in that compound's absorbance at $517 \mathrm{~nm}$ spectrophotometrically with the same instrument as cited above for the determination of polyphenols. Each assay was performed in triplicate with the value of antioxidant activity being expressed as mg equivalents of vitamin E (eq. vit. E) per $100 \mathrm{~g}$ DW.

2.5.2 Antioxidant Capacity in Vivo (Zebrafish Model)

The zebrafish model was used to determine the antioxidant capacity of $C$. microphylla pulp extract in vivo. Wild-type zebrafish of about 4-12 months old were kept in tanks with pure chlorine-free water at a temperature of $28 \pm 1{ }^{\circ} \mathrm{C}$ and equipped with an air-sparging system to maintain oxygen saturation. The fish were raised at a male-to-female ratio of 1:2 and a density of $1 \mathrm{fish} / \mathrm{L}$ water with a photoperiod (16 h of light $/ 8 \mathrm{~h}$ of dark). Larvae of $192 \mathrm{~h}$ post-fertilization were used for the evaluation of toxicity and for the lipid peroxidation assay. 
Lethality is the most commonly used endpoint in the acute-toxicity test, as death is an unambiguous response. Accordingly, in the test, mortality was counted at various concentrations of exposure to piquillin extract to determine the concentration permitting a maximal tolerance (i.e., survival). In the negative control, $100 \%$ of the larvae survived. The cumulative mortality was determined as a function of the logarithm of the concentration, and from this figure the value of the concentration lethal to $50 \%$ of the probands (i.e., the LC50) was determined at a $95 \%$ confidence level. Statistical analysis was performed by the probit regression model, according to the methodology proposed by Spearman Karbar [19].

The antioxidant activity of extracts from the piquillin $(1.44 \mu \mathrm{g}$ eq. $\mathrm{GAE} / \mathrm{mL})$ was evaluated in vivo in the presence of $\mathrm{H}_{2} \mathrm{O}_{2}$ as the oxidizing agent. After three washes with the larval medium [20], the larvae were exposed to $2.4 \% \mathrm{v} / \mathrm{v} \mathrm{H}_{2} \mathrm{O}_{2}$ solution in embryo medium for $16 \mathrm{~h}$ at $28{ }^{\circ} \mathrm{C}$. Lipid peroxidation was determined by monitoring the formation of malondialdehyde (MDA) with TBA, as described by Ohkawa et al. [21]. The results are expressed as the concentration of MDA generated in the sample as reflected in the formation of the MDA-TBA adduct that is measured colorimetrically at $532 \mathrm{~nm}$. Two biological replicates were performed per treatment, with each one involving 40 larvae/well. This procedure was repeated three times, independently.

\subsection{Statistical Analysis}

The results were expressed as the means \pm standard deviation (SD), and the statistical difference between treatments was tested by the one-way analysis of variance (ANOVA). Differences in $p<0.05$ were considered statistically significant.

\section{Results and Discussion}

\subsection{Analysis of Phytonutrients in C. microphylla}

C. microphylla (piquillin) fruit represents a health-promoting food choice, whose nutritional characteristics are listed in Table 1 . The values proved to be in the same order as those published by the USDA National Nutrient Database (2015) for Zizyphus jujuba (Chinese jujube, another species of Rhamnaceae family).

Fruits and berries are generally not considered especially good sources of protein. In contradiction to this misconception, the protein content of piquillin fruit was found to be similar to that of five cultivars of Z. jujuba (4.75\%-6.86\%), but this value was higher than that of $Z$. mistol fruit $(3.06 \pm 0.31 \mathrm{~g} / 100 \mathrm{~g} \mathrm{DW})$, which grows in Northern Argentina [22]. The proteins present in the mesocarp of fleshy fruits tend to be associated with the metabolic processes of the plant, environment and the stress of pathogen exposure. When plants are subjected to different environmental stresses, qualitative and quantitative changes in the protein content occur [23]. This phenomenon could explain the levels of total proteins found in the pulp of the fruit of C. microphylla (4.85\%), originating in the xerophytic Monte in Northern Patagonia, whose values were higher than those reported for $Z$. mauritiana grown in the fertile plains of Bangladesh (i.e., $0.85 \%-1.2 \%$ ) [24].

The nutritive value of the pulp was $3.32 \mathrm{kcal} / \mathrm{g}$ DW,

Table 1 Nutrient composition of the fresh edible portion of $C$. microphylla fruit per $100 \mathrm{~g}$ DW.

\begin{tabular}{ll}
\hline Nutrients & Content $(\mathrm{g} / 100 \mathrm{~g} \mathrm{DW})^{\mathrm{a}}$ \\
\hline Protein & $4.8 \pm 0.2$ \\
Crude fiber & $17.0 \pm 0.8$ \\
Total carbohydrates & $75.0 \pm 2.0$ \\
Lipid & $1.5 \pm 0.5$ \\
Ash & $2.0 \pm 0.1$ \\
\hline
\end{tabular}

\footnotetext{
${ }^{\mathrm{a}}$ The values are the mean $\pm \mathrm{SD}$ of three measurements and are expressed as $\mathrm{g}$ per $100 \mathrm{~g} \mathrm{DW}$.
} 
twice that of Z. rugose $(1.55 \mathrm{kcal} / \mathrm{g}$ DW) [25]. The present investigation revealed that the pulp of the fruit contained a nutritive value in the same range as that of Ziziphus [24]. Indrayan et al. [26] discussed the nutritional benefit of edible plant resources and classified such plants according to the categories of food, fodder and medicinal uses. The aim of the present study was to promote the utilization of wild edible piquillin fruit for uses other than simply food.

\subsection{Soluble Sugar Content}

The soluble sugar content in C. microphylla was $453 \mathrm{mg} / \mathrm{g}, 3.7$ times higher than that of the ripe jujube fruit $(122 \mathrm{mg} / \mathrm{g})$ [27] and 2.5 times the highest total sugar content $(179 \mathrm{mg} / \mathrm{g})$ found in the jujube fruits of cv. Zaytoni [28]. Soluble sugars may either act directly as negative internal signals or as modulators of plant sensitivity, as such also playing a fundamental role in cell responses to stress-induced external signals [29]. Of the total sugar content in the fruit from $C$. microphylla, some $60 \%$ is soluble. This elevated level compared to that of the jujube fruit could have resulted from tolerance to environmental stresses in the Argentine Patagonia or to the colonization of some fungal pathogen. Recent descriptive ecological and agronomic studies have uncovered a strong correlation between soluble sugar concentrations and stress tolerance [30]. Furthermore, the fruits in that southern region of the country tend to exhibit increased contents of reducing sugars during maturity, especially within the later stages, where the glucose and fructose levels become elevated by nearly twofold [3]. The results in present study indicated that the reducing sugars represented some $65 \%(29.61 \pm 2.8 \mathrm{~g}$ glucose eq./100 g DW) of the soluble sugars, a finding in agreement with the increase in sweetness of piquillin fruit upon ripening when the colour becomes mainly brown.

\subsection{Bioactive Phytocompounds}

Table 2 listed the TPCs extracted from $C$. microphylla fruits by three different solvents and illustrated the significant differences $(p<0.05)$ in the TPCs obtained. For example, the total polyphenol content extracted with $70 \%$ aqueous acetone $(2,760$ mg GAE/100 g DW) was more than twice that obtained with the same solvent from Z . mistol fruit (about 1,300 mg/100 g GAE) reported by Cardozo et al. [2] — a Rhamnaceae species grown in Northwestern Argentina. This difference could have resulted from an influence that the altitude and annual precipitation of that region exert over the amount of natural antioxidants [31]. Moreover, it was observed that dimethyl-sulfoxide extracted 1.7 times more total polyphenols than $70 \% \mathrm{v} / \mathrm{v}$ acetone and four times more than methanol (Table 2). Selecting the right solvent affects the amount and rate of polyphenol extraction. Indeed, under the same condition of extraction time and temperature, the solvent used and the chemical properties of the sample matrix are the most influential determinants of the extraction of polyphenols.

\subsection{Individual Phenolic Compounds in Extracts of Piquillin}

Natural phenolics are of interest from many viewpoints (e.g., antioxidant activity, astringency, bitterness, browning action and colour, among others). Table 3 itemized the contents of individual phenolic acids and flavonoids extracted by different solvents. Among the polyphenols analysed, gallic acid was found at a notable concentration of the DMSO extract,

Table 2 TPC of $C$. microphylla fruit obtained with different extraction solvents.

\begin{tabular}{ll}
\hline Solvent & TPC (mg GAE/100 g DW) \\
\hline Acetone $70 \% \mathrm{v} / \mathrm{v}$ & $2,760 \pm 490$ \\
Methanol & $1,140 \pm 110$ \\
Dimethyl sulfoxide $50 \% \mathrm{v} / \mathrm{v}$ & $4,630 \pm 170$ \\
\hline
\end{tabular}


$(2.08 \mathrm{mg} / \mathrm{L})$, but was not detectable in the others. The $p$-coumaric acid was extracted with either methanol or DMSO (though extremely poorly with aqueous acetone), but the content was three times higher in the DMSO extract. Although the ascorbic acid content of three jujube cultivars had been found to be high, this antioxidant remained undetected in this extracts (Table 3). This difference could have resulted from factors influence, such as storage conditions and fruit ripening [32].

Of the flavonoids analyzed, rutin was the principal compound and was extracted by all three solvents (Table 3). Other flavonoids, such as catechin and epicatechin, were extracted by only DMSO. These results are in accordance with the TPC determination by the Folin-Ciocalteu method, in which the solvent $50 \% \mathrm{v} / \mathrm{v}$ aqueous DMSO extracted is of greater quantities of the phenolic compounds (Table 2).

\subsection{Anthocyanin Content}

Since the content of extracted polyphenols and anthocyanins has been found to vary with the use of different solvents and extraction times, the method of quantifying the total monomeric anthocyanin content has been accordingly standardized [33]. In the present work, the content of anthocyanin in acetone extracts was $111 \pm 18 \mathrm{~g} \mathrm{C} 3 \mathrm{GE} / 100 \mathrm{~g} \mathrm{DW}$, while the amount extracted by methanol was $192 \pm 25 \mathrm{~g} \mathrm{C} 3 \mathrm{GE} / 100 \mathrm{~g}$
DW. The antioxidant, however, was refractory to extraction by DMSO. This difference might have resulted from the failure of the method to measure anthocyanin pigments that have become polymerized [16]. Anthocyanin complexes or copigmentation reactions with the simpler anthocyanins can occur through intra- or inter-molecular interactions with an organic acid, an aromatic acyl group or a flavonoid [34]. Since the results indicated that the DMSO extract contained the organic acids $p$-coumarate and gallate (Table 3 ), those components could accordingly have interacted with the anthocyanins that were present.

\subsection{Antioxidant Potentials of C. microphylla Fruit}

\subsubsection{In Vitro Antioxidant Activities with DPPH}

Chemical assays in vitro, such as the DPPH test, constitute a robust method to quickly screen for antioxidant or antiradical activities in extracts or pure compounds. Fig. 1 depicted the DPPH-scavenging activities exhibited by different solvent extracts from C. microphylla. All three extracts examined were significantly active as free radical-scavengers. Among the three, the acetone and DMSO extracts exhibited statistically equivalent activities (19.4 mg and $17.1 \mathrm{mg}$ eq. vit. E/g DW, respectively), but both extracts were significantly more active than the methanol preparation (4.85 $\mathrm{mg}$ eq. vit. E/g DW). Although a

Table 3 Composition of individual phenols obtained from $C$. microphylla fruit by extraction with different solvents and expressed as $\mathbf{m g} / \mathrm{L}$.

\begin{tabular}{llll}
\hline Polyphenols $(\mathrm{mg} / \mathrm{L})$ & Acetone $70 \% \mathrm{v} / \mathrm{v}$ & Methanol & DMSO 50\% v/v \\
\hline Ascorbic acid & $\mathrm{ND}$ & $\mathrm{ND}$ & $\mathrm{ND}$ \\
Caffeic acid & $\mathrm{ND}$ & $\mathrm{ND}$ & $\mathrm{ND}$ \\
Chlorogenic acid & $<3.5$ & $<3.5$ & $<3.5$ \\
$p$-coumaric acid & $<1.0$ & 4.34 & 12.0 \\
Ferulic acid & $\mathrm{ND}$ & $\mathrm{ND}$ & $\mathrm{ND}$ \\
Gallic acid & $\mathrm{ND}$ & $\mathrm{ND}$ & 2.08 \\
Rutin & 12.0 & 8.23 & 8.80 \\
Catechin & $<1.5$ & $<1.5$ & 3.30 \\
Epicatechin & $<2.0$ & $<2.0$ & 6.36 \\
Phloridzin HPLC & $\mathrm{ND}$ & $\mathrm{ND}$ & $\mathrm{ND}$ \\
HMF & $\mathrm{ND}$ & $\mathrm{ND}$ & $\mathrm{ND}$ \\
\hline
\end{tabular}

ND: not detected; HMF: 5-(hydroxymethyl)-2-furfural. 


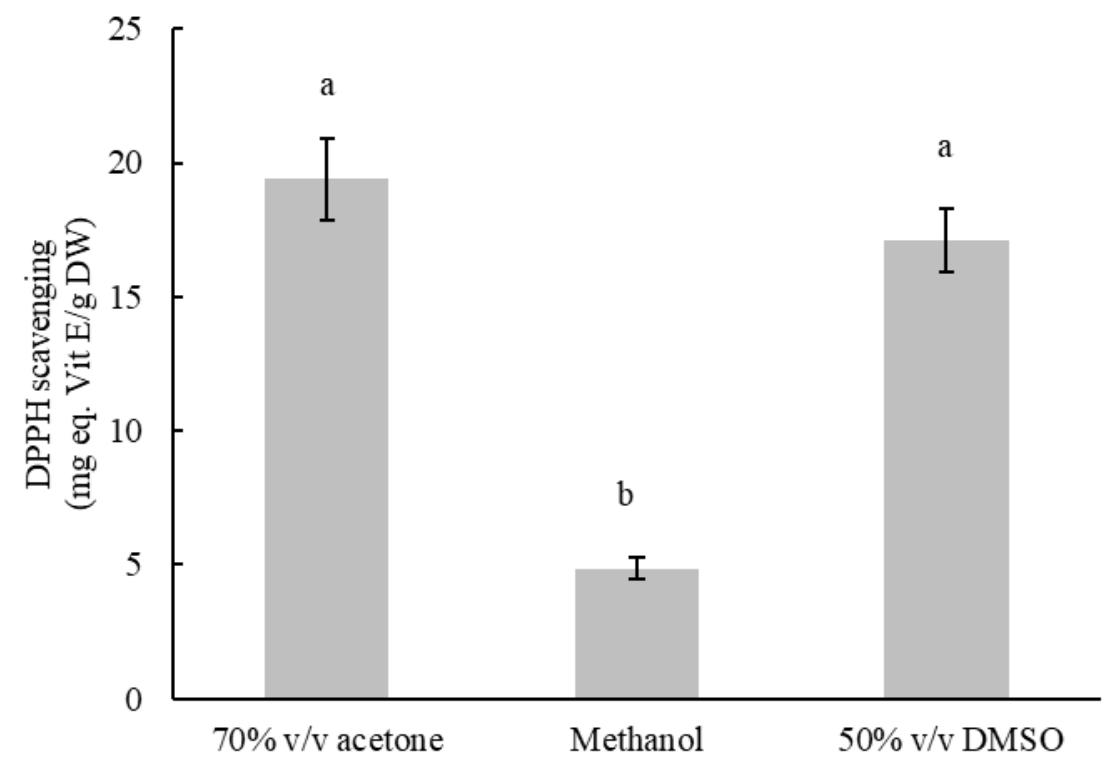

Fig. 1 Antioxidant activity of piquillin fruit extracted by different solvents.

The vertical bars represent $\mathrm{SD}(n=3)$.

In the figure, the free radical-scavenging activity in $\mathrm{mg}$ equivalents of vitamin $\mathrm{E}$ ( $\mathrm{mg}$ eq. vit. E) per DW, determined with $0.5 \mathrm{mM}$ $\mathrm{DPPH}$ as the target radical, is plotted on the ordinate for each of the extracts indicated on the abscissa.

direct correlation between the antioxidant capacity and the TPC of extracts has been reported [35], the strict relationship could not be demonstrated in the present work (Table 3).

Quercetin seems to be the most powerful flavonoid for protecting the body from reactive oxygen species produced during normal oxygen metabolism or from those radicals that are induced by external damage [36]. Of the flavonoids measured in the three piquillin fruit extracts by reverse-phase high-performance liquid chromatography (Table 3), rutin was the principal one in all the samples, and particularly so in the acetone extract at a level about 1.4 times higher than those of the other two preparations. In contrast, catechin and epicatechin were detected in only the DMSO extract $(3.30 \mathrm{mg} / \mathrm{L}$ and $6.36 \mathrm{mg} / \mathrm{L}$, respectively). Iacopini et al. [37] had found that quercetin exhibited the highest antioxidant activity with respect to the scavenging of DPPH free radicals. Catechin and epicatechin were weaker in DPPH scavenging than quercetin. These authors also demonstrated that the antioxidant activity of extracts is not dependent simply on the TPC, but that phenolic compounds can act in synergy or antagonism - as opposed to straight additivity - to affect the total activity of mixtures. Thus, such conceivable interactions among the phenolic compounds could be considered an explanation for why no simple relationship was found to exist between the antioxidant activity and TPC (Table 2) of the authors' extracts. Nevertheless, all of these results indicated that $70 \% \mathrm{v} / \mathrm{v}$ aqueous acetone was likely to be a better solvent for the extraction of antioxidants from $C$. microphylla for the purpose of evaluating DPPH-scavenging activity.

\subsubsection{In Vivo Antioxidant Activities}

The in vivo inhibition of lipid peroxidation capacity of acetone-derived extract was tested using the zebrafish larvae model. This capacity was evaluated as the protective effect of extract to the lipid peroxidation induced by hydrogen peroxide in the zebrafish larvae.

In order to determine the antioxidant activity in vivo of extracts, dose-response curves were conducted to determine the working range and the LC50 for the extract with the highest antioxidant activity (i.e., acetone:water $=70: 30$ ). Since toxicity of the extracts 
may be caused by, among other substances, the presence of low-molecular-weight polyphenols [38], it was determined the effect of extract dosage on zebrafish larval lethality. Fig. 2 plotted the accumulated percentage of dead larvae as a function of the polyphenol concentration of the extract. The LC50, determined by the probit, according to the method of Spearman Karbar [19], was $2.87 \pm 0.4 \mu \mathrm{g}$ $\mathrm{GAE} / \mathrm{mL}$.

The percentages of inhibition of peroxidation lipid were shown in Fig. 3. Since the metabolic product of lipid peroxidation in vertebrates is MDA, this compound was assayed after a major oxidative stress on those larvae. It was observed that the MDA levels in the zebrafish extracts exposed to $2.4 \% \mathrm{v} / \mathrm{v} \mathrm{H}_{2} \mathrm{O}_{2}$ increased significantly $(30 \% \pm 3.8)$ compared to those of the control group, indicating that a large amount of lipid peroxidation had occurred in the treated larvae. The basal MDA concentration quantified in the fish larvae was $1.34 \mu \mathrm{M}$ (i.e., the $100 \%$ level in Fig. 3), a value within the range reported by other authors in this same biological model [39]. When the larvae were incubated in the presence of the oxidant plus a phenolic extract from the piquillin drupes (containing $1.44 \mu \mathrm{g}$ eq. GAE/mL), a marked inhibition of lipid peroxidation resulted-i.e., down to $89 \% \pm 0.8$ of that control value $(p<0.01)$. Thus, an extract of the piquillin fruit was capable of completely protecting those $192 \mathrm{~h}$ post-fertilization zebrafish larvae from oxidative stress. In fact, the degree of oxidation in the presence of the extract was even significantly less than the control value or the level with the extract alone in the absence of oxidative stress (i.e., $1.33 \mu \mathrm{M}$ ). This added elimination of basal plus induced peroxidation would appear to indicate some form of synergistic interaction between the polyphenol-containing extracts and the larval endogenous antioxidation system, but one that apparently engages only within

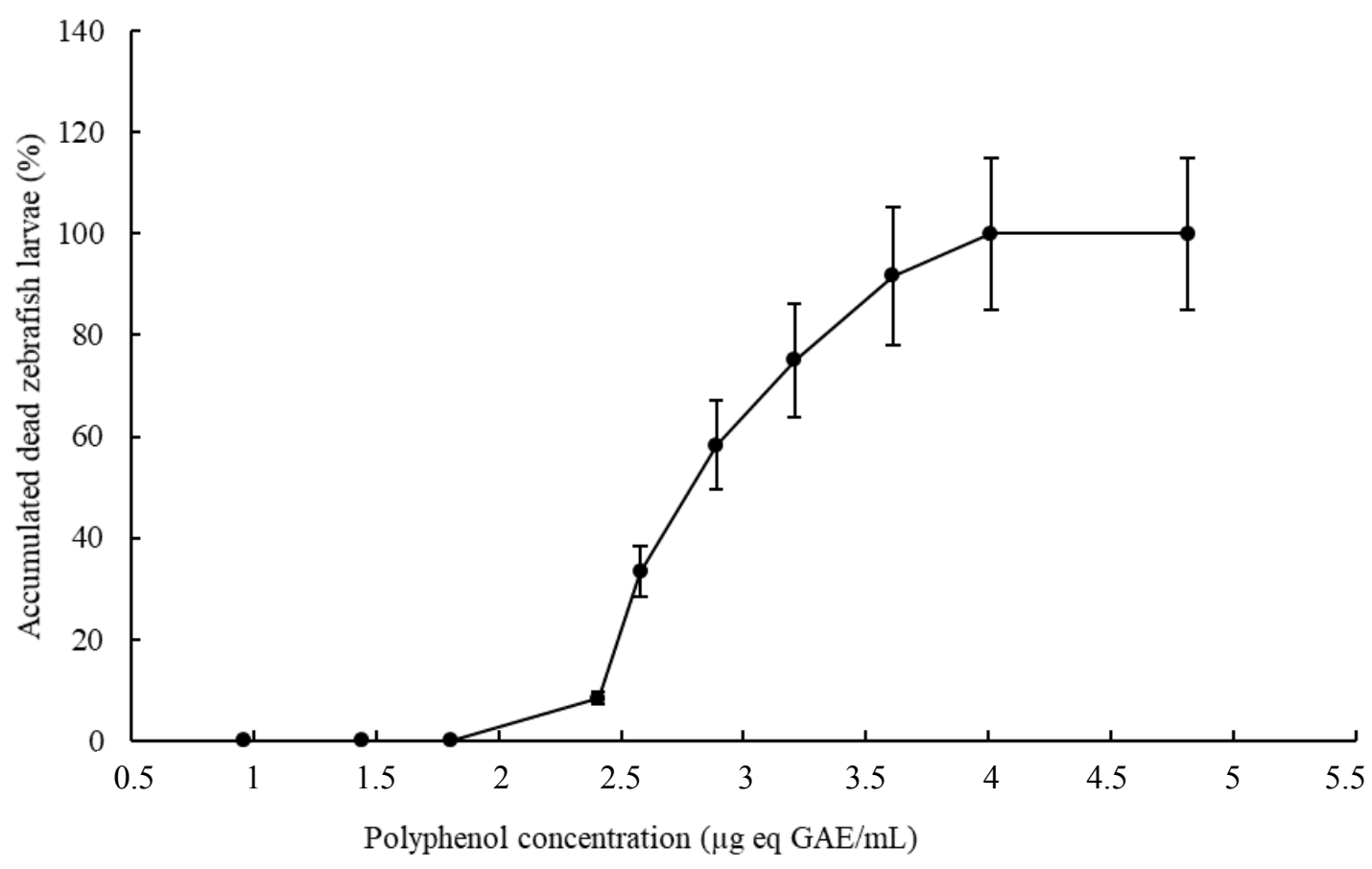

Fig. 2 Dose-response curve of the toxicity of polyphenolic compounds in $5 \%$ (v/v) dimethyl sulfoxide to zebrafish larvae after a 96 h exposure.

In the figure, the percent of dead zebrafish larvae is plotted on the ordinate as a function of polyphenol concentration in $\mu \mathrm{g}$ of gallic acid equivalents (eq. GAE) on the abscissa.

Each point represents the mean value of triplicate readings. 


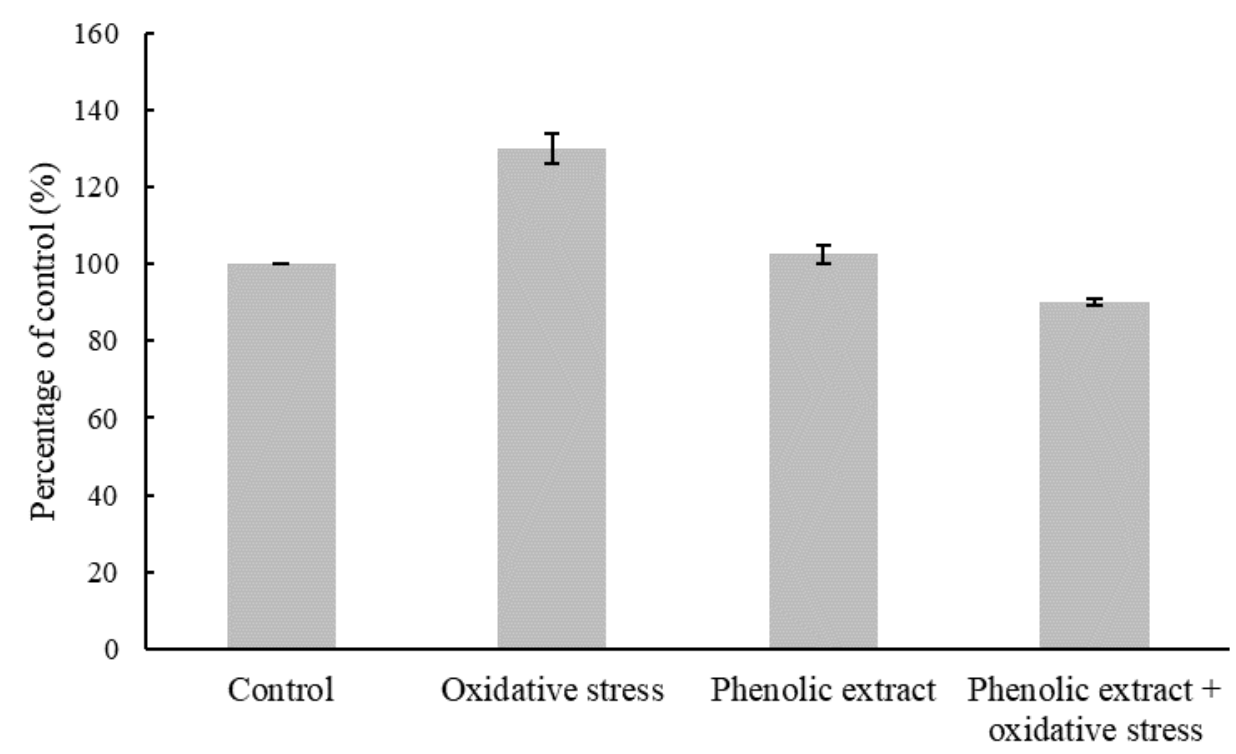

Fig. 3 Changes in MDA content in zebrafish (D. rerio) larvae (192 h post-fertilization) upon exposure to oxidative stress $\left(2.4 \%(\mathrm{v} / \mathrm{v}) \mathrm{H}_{2} \mathrm{O}_{2}\right)$ in the presence of a phenolic extract $(1.44 \mu \mathrm{g} \mathrm{eq.} \mathrm{GAE} / \mathrm{mL})$.

In the figure, the percent MDA concentration of the control group, as a measure of lipid peroxidation, is plotted on the ordinate for each of the experimental conditions indicated on the abscissa.

The values are means \pm SD indicated by the brackets $(n=3)$.

The MDA content in the control group ( $100 \%$ value on the ordinate) was $1.34 \mu \mathrm{M}$.

the context of oxidation stress, as the basal MDA levels were equivalent to those of the extract alone in the absence of added peroxide.

To correct for excessive false-positive results, stringent endpoint criteria must be adopted. In general, for a realistic assessment of the presence or absence of noxious substances, LC50 values should be below 100 $\mu \mathrm{g} / \mathrm{mL}$ for mixtures and below $25 \mu \mathrm{M}$ for pure compounds [40]. The results in present study demonstrated that a concentration polyphenols 70 times lower than the LC50 of the active compound (i.e., $1.44 \mu \mathrm{g}$ eq. $\mathrm{GAE} / \mathrm{mL}$ ) was able to inhibit lipid oxidation by up to $40 \%$.

\section{Conclusions}

The present study demonstrated how the nutritional composition of C. microphylla can be used for different purposes. Because the fruit is rich in energy and secondary metabolites, it could be considered a product for human nutrition with potential health benefits. It is proposed the extraction methodology and the most adequate analytical technique to achieve the highest biological activity of the secondary metabolites from piquillin fruits. Moreover, the results of the biological characterization reported here indicated that the drupes of C. microphylla possess a significant antioxidant activity in vitro and in vivo. Therefore, piquillin fruit is a promising source of antioxidant molecules that could exert a preventive effect against oxidative stress-associated disorders and might be used as natural additive for food. In addition, the information generated in this work could lengthen the list of bioactive compounds for current scientific evaluation. Further studies are needed to investigate the bioavailability and mechanism of action of the compounds included in piquillin fruits.

\section{Acknowledgments}

Financial support from the Consejo Nacional de Investigaciones Científicas y Técnicas (CONICET), Universidad Nacional de Río Negro is gratefully acknowledged. Sincere thanks also expressed to Dr. Donald F. Haggerty, a retired academic career 

a Native Species of Patagonia Argentina

investigator and native English speaker for editing the final version of the manuscript.

\section{References}

[1] Barboza, G. E., Cantero, J., Núñez, C., Pacciaroni, A., and Espinar, L. A. 2009. "Medicinal Plants: A General Review and a Phytochemical and Ethnopharmacological Screening of the Native Argentine Flora." Kurtziana 34 (1-2): 7-365.

[2] Cardozo, M. L., Ordoñez, R. M., Alberto, M. R., Zampini, I. C., and Isla, M. I. 2011. "Antioxidant and Anti-inflammatory Activity Characterization and Genotoxicity Evaluation of Ziziphus mistol Ripe Berries, Exotic Argentinean Fruit.” Food Research International 44 (7): 2063-71.

[3] Guo, S., Duan, J., Qian, D., Tang, Y., Wu, D., Su, S., Wuang, H., and Zhao, Y. 2015. "Content Variations of Triterpenic Acid, Nucleoside, Nucleobase and Sugar in Jujube (Ziziphus jujuba) Fruit during Ripening." Food Chemistry 167: 468-74.

[4] Conti, J., Belardinelli, L., Utterback, D., and Curtis, A. 1995. "Endogenous Adenosine Is an Antiarrhythmic Agent." Circulation 91: 1761-7.

[5] Virag, L., and Szabo, C. 2001. "Purines Inhibit Poly (ADP-Ribose) Polymerase Activation and Modulate Oxidant-Induced Cell Death.” FASEB Journal 15 (1): 99-107.

[6] Schmidt, A. P., Lara, D. R., De Faria Maraschin, J., Da Silveira Perla, A., and Souza, D. O. 2000. "Guanosine and GMP Prevent Seizures Induced by Quinolinic Acid in Mice." Brain Research 864 (1): 40-3.

[7] Plastina, P., Bonofiglio, D., Vizza, D., Fazio, A., Rovito, D., Giordano, C., Barone, I., Catalano, S., and Gabriele, B. 2012. "Identification of Bioactive Constituents of Ziziphus jujube Fruit Extracts Exerting Antiproliferative and Apoptotic Effects in Human Breast Cancer Cells." Journal of Ethnopharmacology 140 (2): 325-32.

[8] Çelik, S. E., Özyürek, M., Güçlü, K., and Apak, R. 2010. "Solvent Effects on the Antioxidant Capacity of Lipophilic and Hydrophilic Antioxidants Measured by CUPRAC, ABTS/Persulphate and FRAP Methods." Talanta 81 (4): 1300-9.

[9] Marwah, R. G., Fatope, M. O., Al Mahrooqi, R., Varma, G. B., Al Abadi, H., and Al-Burtamani, S. K. S. 2007. "Antioxidant Capacity of Some Edible and Wound Healing Plants in Oman.” Food Chemistry 101 (2): 465-70.

[10] Pereira Lima, G. P., Vianello, F., Corrêa, C. R., Da Silva Campos, R. A., and Borguini, M. G. 2014. "Polyphenols in Fruits and Vegetables and Its Effect on Human Health." Food and Nutrition Sciences 5: 1065-82.
[11] Zon, L. I., and Peterson, R. T. 2005. "In Vivo Drug Discovery in the Zebrafish." Nature Reviews Drug Discovery 4 (1): 35-44.

[12] Vilela, A. E., González-Paleo, L., and Ravetta, D. A. 2011. "Secondary Metabolites of Woody Plants from Arid Lands: Mechanisms Involved, Functions and Uses." Asociación Argentina de Ecología, Ecología Austral 21: 317-27.

[13] Association of Official Analytical Chemists (AOAC) International. 1990. Official Methods for Analysis of the Association of Official Analytical Chemists. 15th ed.. Washington, D.C.: AOAC.

[14] Dubois, M., Gilles, K. A., Hamilton, J. K., Rebers, P., and Smith, F. 1956. "Colorimetric Method for Determination of Sugars and Related Substances." Analytical Chemistry 28 (3): 350-6.

[15] Somogyi, M. 1952. "Determination of Reducing Sugars by Nelson-Somogyi Method." Journal of Biological Chemistry 200: 245.

[16] Singleton, V. L., Orthofer, R., and Lamuela-Raventós, R. M. 1999. "Analysis of Total Phenols and Other Oxidation Substrates and Antioxidants by Means of Folin-Ciocalteu Reagent." Methods in Enzymology 299: 152-78.

[17] Lee, J., Durst, R. W., and Wrolstad, R. E. 2005. "Determination of Total Monomeric Anthocyanin Pigment Content of Fruit Juices, Beverages, Natural Colorants and Wines by the $\mathrm{pH}$ Differential Method: Collaborative Study." Journal of AOAC International 88 (5): 1269-78.

[18] Brand-Williams, W., Cuvelier, M. E., and Berset, C. 1995. "Use of a Free Radical Method to Evaluate Antioxidant Activity." Food Science Technology 28: 25-30.

[19] Hamilton, M. A., Russo, R. C., and Thurston, R. V. 1977. "Trimmed Spearman Karbar Method for Estimating Lethal Concentrations in Toxicity Bioassays." Environ. Sci. Technol. 11: 714-8.

[20] Westerfield, M. 2000. The Zebrafish Book: A Guide for the Laboratory Use of Zebrafish (Danio rerio). 4th ed.. Eugene, OR: University of Oregon Press.

[21] Ohkawa, H., Ohishi, N., and Yagi, K. 1979. "Assay for Lipid Peroxides in Animal Tissues by Thiobarbituric Acid Reaction." Analytical Biochemistry 95 (2): 351-8.

[22] Li, J. W., Fan, L. P., Ding, S. D., and Ding, X. L. 2007. "Nutritional Composition of Five Cultivars of Chinese Jujube." Food Chemistry 103 (2): 454-60.

[23] Chan, Z. 2013. "Proteomic Responses of Fruits to Environmental Stresses." Frontiers in Plant Science 3: 311.

[24] Talukdar, M. I., Rahman, S. S., Akhtaruzzaman, M., and Samad, M. A. 2014. "A Comparative Study on the Nutritional Quality of Five Varieties of Bangladesh Jujubes (Zizyphus mauritiana)." American Journal of Nutrition and Food Science 32: 36. 


\section{a Native Species of Patagonia Argentina}

[25] Krishnamurthy, S. R., and Sarala, P. 2012. "Determination of Nutritive Value of Ziziphus rugosa Lamk.: A Famine Edible Fruit and Medicinal Plant of Western Ghats.” Indian Journal of Natural Products and Resources 3 (1): 20-7.

[26] Indrayan, A. K., Sharma, S., Durgapal, D., Kumar, N., and Kumar, M. 2005. "Determination of Nutritive Value and Analysis of Mineral Elements for Some Medicinally Valued Plants from Uttaranchal." Current Science 89: $1252-5$

[27] Lu, H., Lou, H., Zheng, H., Hu, Y., and Li, Y. 2012. "Nondestructive Evaluation of Quality Changes and the Optimum Time for Harvesting during Jujube (Zizyphus jujuba Mill. cv. Changhong) Fruits Development." Food and Bioprocess Technology 5 (6): 2586-95.

[28] Abbas, M. E. F., and Fandi, B. S. 2002. "Respiration Rate, Ethylene Production and Biochemical Changes during Fruit Development and Maturation of Jujube (Ziziphus mauritiana Lamk)." Journal of the Science of Food and Agriculture 82 (13): 1472-6.

[29] Rosa, M., Prado, C., Podazza, G., Interdonato, R., González, J. A., Hilal, M., and Prado, F. E. 2009. "Soluble Sugars: Metabolism, Sensing and Abiotic Stress: A Complex Network in the Life of Plants." Plant Signaling \& Behavior 4 (5): 388-93.

[30] Pareek, S. 2013. "Nutritional Composition of Jujube Fruit." Emirates Journal of Food and Agriculture 25 (6): 463.

[31] Sun, L., Zhang, J., Lu, X., Zhang, L., and Zhang, Y. 2011. "Evaluation to the Antioxidant Activity of Total Flavonoids Extract from Persimmon (Diospyros kaki L.) Leaves." Food and Chemical Toxicology 49 (10): 2689-96.

[32] Zhang, C., Huang, J., and Li, X. 2016. "Transcriptomic Analysis Reveals the Metabolic Mechanism of L-Ascorbic Acid in Ziziphus jujuba Mill." Frontiers in
Plant Science 7 (122): 1-22.

[33] Giusti, M. M., and Wrolstad, R. E. 2001. "Characterization and Measurement of Anthocyanins by UV-Visible Spectroscopy." Current Protocols in Food Analytical Chemistry. doi:10.1002/0471142913.faf0 102s00.

[34] Eiro, M. J., and Heinonen, M. 2002. “Anthocyanin Color Behavior and Stability during Storage: Effect of Intermolecular Copigmentation." Journal of Agricultural and Food Chemistry 50 (25): 7461-6.

[35] Yang, C. M., Lee, C. N., and Chou, C. H. 2002. "Effects of Three Allelopathic Phenolics on Chlorophyll Accumulation of Rice (Oryza sativa) Seedlings: I. Inhibition of Supply-Orientation." Botanical Bulletin of Academia Sinica 43: 299-304.

[36] De Groot, H. 1994. "Reactive Oxygen Species in Tissue Injury." Hepato-Gastroenterology 41 (4): 328-32.

[37] Iacopini, P., Baldi, M., Storchi, P., and Sebastiani, L. 2008. "Catechin, Epicatechin, Quercetin, Rutin and Resveratrol in Red Grape: Content, in Vitro Antioxidant Activity and Interactions." Journal of Food Composition and Analysis 21 (8): 589-98.

[38] Fiorentino, A., Gentili, A., Isidori, M., Monaco, P., Nardelli, A., Parrella, A., and Temussi, F. 2003. "Environmental Effects Caused by Olive Mill Wastewaters: Toxicity Comparison of Low-Molecular-Weight Phenol Components." Journal of Agricultural and Food Chemistry 51 (4): 1005-9.

[39] Zhang, W., Liu, W., Zhang, J., Zhao, H., Zhang, Y., Quan, X., and Jin, Y. 2012. "Characterization of Acute Toxicity, Genotoxicity and Oxidative Stress Posed by Textile Effluent on Zebrafish." Journal of Environmental Sciences 24 (11): 2019-27.

[40] Cos, P., Vlietinck, A. J., Berghe, D. V., and Maes, L. 2006. "Anti-infective Potential of Natural Products: How to Develop a Stronger in Vitro 'Proof-of-Concept'." Journal of Ethnopharmacology 106 (3): 290-302. 